\title{
Risk Reduction of Breast Cancer by Childbirth, Breastfeeding, and Their Interaction in Korean Women: Heterogeneous Effects Across Menopausal Status, Hormone Receptor Status, and Pathological Subtypes
}

\author{
Seok Hun Jeong ${ }^{1,2}$, Yoonsuk An ${ }^{1,2}$, Ji-Yeob Choi ${ }^{1,2,3}$, Boyoung Park ${ }^{4,5}$, Daehee Kang ${ }^{1,2,3}$, Min Hyuk Lee ${ }^{6}$, Wonshik Han ${ }^{2,7}$, \\ Dong-Young Noh ${ }^{2,7}$, Keun-Young Yoo ${ }^{1,8}$, Sue K. Park ${ }^{1,2,3}$ \\ ${ }^{1}$ Department of Preventive Medicine, Seoul National University College of Medicine, Seoul; ${ }^{2}$ Cancer Research Institute, Seoul National University, \\ Seoul; ${ }^{3}$ Department of Biomedical Science, Seoul National University Graduate School, Seoul; ${ }^{4}$ National Cancer Center Graduate School of Cancer \\ Science and Policy, Goyang; ${ }^{5}$ National Cancer Control Institute, National Cancer Center, Goyang; ${ }^{6}$ Department of Surgery, Soonchunhyang \\ University College of Medicine, Seoul; ${ }^{7}$ Department of Surgery, Seoul National University Hospital, Seoul National University College of Medicine, \\ Seoul; ${ }^{8}$ Armed Forces Capital Hospital, Seongnam, Korea
}

Objectives: The purpose of this study was to examine the associations of childbirth, breastfeeding, and their interaction with breast cancer $(B C)$ risk reduction, and to evaluate the heterogeneity in the $B C$ risk reduction effects of these factors by menopause, hormone receptor (HR) status, and pathological subtype.

Methods: BC patients aged 40+ from the Korean Breast Cancer Registry in 2004-2012 and controls from the Health Examinee cohort participants were included in this study after 1:1 matching (12 889 pairs) by age and enrollment year. BC risk according to childbirth, breastfeeding, and their interaction was calculated in logistic regression models using odds ratios (ORs) and 95\% confidence intervals (Cls). Results: $\mathrm{BC}$ risk decreased with childbirth (3+ childbirths relative to 1 childbirth: $\mathrm{OR}, 0.66 ; 95 \% \mathrm{Cl}, 0.56$ to 0.78 and $\mathrm{OR}, 0.80 ; 95 \% \mathrm{Cl}$, 0.68 to 0.95 in postmenopausal and premenopausal women, respectively); and the degree of risk reduction by the number of children was heterogeneous according to menopausal status ( $p$-heterogeneity $=0.04$ ), HR status ( $p$-heterogeneity $<0.001$ ), and pathological subtype ( $p$-heterogeneity $<0.001$ ); whereas breastfeeding for 1-12 months showed a heterogeneous association with $B C$ risk according to menopausal status, with risk reduction only in premenopausal women ( $p$-heterogeneity<0.05). The combination of 2 more childbirths and breastfeeding for $\geq 13$ months had a much stronger $\mathrm{BC}$ risk reduction of $49 \%$ (OR, $0.51 ; 95 \% \mathrm{Cl}, 0.45$ to 0.58 ).

Conclusions: This study suggests that the combination of longer breastfeeding and more childbirths reduces $\mathrm{BC}$ risk more strongly, and that women who experience both 2 or more childbirths and breastfeed for $\geq 13$ months can reduce their BC risk by about $50 \%$.

Key words: Breast cancer, Childbirth, Breastfeeding, Risk, Reduction

Received: September 25, 2017 Accepted: November 10, 2017

Corresponding author: Sue K. Park, MD, PhD

103 Daehak-ro, Jongno-gu, Seoul 03080, Korea

Tel: +82-2-740-8338, Fax: +82-2-747-4830

E-mail: suepark@snu.ac.kr

This is an Open Access article distributed under the terms of the Creative Commons Attribution Non-Commercial License (http://creativecommons.org/licenses/by$\mathrm{nc} / 4.0 / /$ which permits unrestricted non-commercial use, distribution, and reproduction in any medium, provided the original work is properly cited.

\section{INTRODUCTION}

Giving birth plays an important role in reducing the risk of breast cancer (BC) in women [1-3]. In addition, breastfeeding, which is inseparable from giving birth, is considered to have a risk reduction effect, although controversies exist [3-8].

Pregnancy and lactation prevent BC through the following 2 major mechanisms. The first is promoting the differentiation 
of mammary cells $[9,10]$. In parous women, the proportion of differentiated terminal ductal lobular tissues is higher than in nulliparous women, and the risk of malignancy is lower, because undifferentiated breast tissues have a higher risk of malignant transformation than differentiated breast tissues. Breastfeeding triggers a continuation of the cell differentiation process and prolongs the time during which the tissue is in a mature state $[11,12]$. The second mechanism is the reduction of lifetime estrogen exposure. According to the estrogen hypothesis, higher estrogen exposure increases the risk of $B C$ [13] in comparison to women in whom ovulatory cycles were attenuated due to pregnancy and lactation $[14,15]$.

Prior meta-analyses have failed to demonstrate a dose-response relationship of breastfeeding on $\mathrm{BC}$ risk due to high heterogeneity among studies (>70\%), a small number of cohort studies (only 3 ), and non-significant dose-response relationship in meta-analysis of cohort studies $[8,16]$. In addition, the combined influence of the number of childbirths and breastfeeding period on the risk of $\mathrm{BC}$ has not been clarified in previous epidemiological studies. The reason for this is that women with 4 or more childbirths and longer breastfeeding periods (over 25 months) comprise less than 5 and $10 \%$ of the total population, respectively. Furthermore, since the risk of $\mathrm{BC}$ by reproductive risk factors depends on menopausal status, the analysis should be stratified by menopausal status. Therefore, larger study populations must be analyzed to identify a dose-response relationship. Furthermore, since the number of childbirths and the breastfeeding period are biologically related, the duration of breastfeeding increases as the number of childbirths increases. In most studies, due to limited sample sizes, it is difficult to distinguish between women who are not breastfed and women who are not breastfed among women who have had one or more childbirths. Because it is known that both factors reduce $B C$ risk, the presence of both a longer breastfeeding duration and more childbirths may exert a combined effect. A previous study was conducted of the interaction of these 2 factors on the risk of ovarian cancer [17], but their interaction with $B C$ risk has not been confirmed, due to various complex situations causing limitations in statistical power.

It has recently been established that $\mathrm{BC}$ has different clinical courses depending on the hormone receptor (HR) status, classified in terms of estrogen and progesterone receptors, the HER2-receptor, and/or Ki-67 levels, and the pathological subtype of cancer. We hypothesized that the protective effect of breastfeeding on $\mathrm{BC}$ risk would be different depending on the number of childbirths, and therefore we investigated whether there was a synergistic effect between parity and breastfeeding. We designed a population-based case-control study using the Nationwide Breast Cancer Registry and woman participants in large-scale population-based health screening programs.

The aims of this case-control study were: 1) to identify the main effects of the number of childbirths and breastfeeding period on $\mathrm{BC}$ risk reduction, while sufficiently controlling for confounding factors; 2 ) to determine whether women with a combined experience of more childbirths and longer breastfeeding duration had a more strongly reduced $B C$ risk than those with exposure to a single protective factor; and 3) to investigate the heterogeneity of the associations of childbirth, breastfeeding, and their combination with $\mathrm{BC}$ risk across menopausal status, HR status, and pathological subtypes.

\section{METHODS}

\section{Study Subjects}

The Korea Breast Cancer Study has a mixed design of a largescale population-based case-control study and cohort study, and is composed of man and woman BC patients from throughout the nation and community-based healthy controls from 2004 to 2012. The eligible BC cases were obtained from the database of the Korean National Breast Cancer Registry, maintained by the Korean Breast Cancer Society (KBCS) [18]. In brief, the KBCS has conducted a nationwide $B C$ registry for man and woman $B C$ patients diagnosed at 102 general hospitals since 1996, and the registration data include more than $95 \%$ of new breast cancer patients in Korea. The BC cases included in this study were woman BC patients aged 40-74 who were registered between 2004 and 2012. We excluded individuals with missing data about both the number of childbirths and the breastfeeding period. The eligible controls were obtained from the database of the Health Examinees Study (HEXA) [19]. In brief, the HEXA is a population-based cohort study of health screening recipients who receive biennial health screenings covered by the National Health Insurance Service, and is one of the cohort studies of the Korean Genome and Epidemiology Study conducted by the Korea Centers for Disease Control and Prevention. The control subjects in this study were defined as healthy women aged between 40 and 74 with no diagnosis of $B C$ who were registered from 2004 to 2012 ( $n=101$ 017). We excluded women with missing data about both the number of childbirths and the breastfeeding 
period. We matched cases and controls 1:1, according to age and enrollment period, which was divided into 3 categories; 2004-2006, 2007-2009, and 2010-2012. Finally, a total of 25 778 case-control sets (12 889 pairs) were selected as study subjects.

\section{Data Collection and Management}

Information about age, family history of BC among first-degree and second-degree relatives, age at menarche, pregnancy history, age at the first full-term pregnancy, parity and the number of childbirths, periods of breastfeeding, oral contraceptive (OC) use, and hormone replacement therapy use was collected by personal interviews.

\section{Statistical Analysis}

Differences between BC cases and controls in the frequencies of general characteristics, such as family history, number of childbirths, breastfeeding experience, $\mathrm{OC}$ use, body mass index (BMI), age at menarche, and age at first full-term pregnancy, were tested using the chi-square test. The number of childbirths was classified as 0 (nulliparous women), 1, 2, and $3+$. Breastfeeding duration was classified as 0 (never breastfeeding), 1-12, 13-24, and 25+ months.

The $B C$ risk associated with parity, number of childbirths, breastfeeding duration, and the combination of the number of childbirths and breastfeeding duration in the entire sample was estimated using conditional logistic regression models with odds ratios (ORs) and 95\% confidence intervals (Cls), stratified by age and enrollment year, and additionally adjusted for BC family history, age at menarche, number of childbirths (for breastfeeding and its duration), breastfeeding duration (for parity and number of childbirths), OC use, and BMI. The $\mathrm{BC}$ risk associated with these risk factors in premenopausal and postmenopausal women was estimated in unconditional logistic regression models, stratified by age and enrollment year and adjusted for same covariates, because the matching pair was broken. Moreover, we classified the $\mathrm{BC}$ patients according to HR status (HR+ or HR- BC cases) and pathologic subtype (such as the luminal A, luminal B, HER2enriched, and triple-negative $B C$ [TNBC] subtypes); and analyzed the $B C$ risk associated with childbirth and breastfeeding duration for each population subset and the total control population in unconditional logistic regression models, accounting for the variability of subgroup-specific OR values across HR and pathological types. To evaluate differences in the associa- tion of individual factors (number of childbirths and breastfeeding duration) and their combination with $\mathrm{BC}$ risk according to menopausal status, HR status, and pathological subtype, the heterogeneity across menopausal status, HR status, and pathological subtype was tested using the Cochran $\mathrm{Q}$ test at a significance level of $p<0.05$ and quantified by the $I^{2}$ statistic, with an $1^{2}$ of $50 \%$ considered to be evidence of substantial heterogeneity [20]. Most statistical analyses, except $p$-heterogeneity analyses, were performed in SAS version 9.4. (SAS Institute Inc., Cary, NC, USA). The 1:1 matching of cases and controls was made using the G-match algorithm [21] of SAS 9.4. The Cochran $\mathrm{Q}$ test was performed in Stata version 14 (StataCorp, College Station, TX, USA).

\section{Ethics approval and consent to participate}

This study was approved by the institutional review board (IRB) of Seoul National University Hospital Biomedical Research Institue (IRB no., 0909-048-295).

\section{RESULTS}

The baseline characteristics of the 25778 women in the study are described in Supplemental Table 1. Statistically significant differences were found in most of the known risk factors, such as a family history of BC, childbirth, breastfeeding, OC use, high BMI, and late age of the first full-term pregnancy; but the distribution of menarche age was not significantly different between cases and controls.

The $B C$ risk by individual factors, such as total childbirths and total breastfeeding duration, premenopausal and postmenopausal status, HR status, and pathological subtype is shown in Tables 1-3 and Supplemental Figures 1-3. The BC risk of nulliparous women was higher than that of parous women, especially in postmenopausal women $(\mathrm{OR}, 1.72 ; 95 \% \mathrm{Cl}, 1.18$ to 2.50). This effect was stronger for $\mathrm{HR}+\mathrm{BC}(\mathrm{OR}, 2.06 ; 95 \% \mathrm{Cl}$, 1.43 to 3.00$)$ and luminal $A B C(O R, 2.15 ; 95 \% \mathrm{Cl}, 1.46$ to 3.18$)$ (data not shown). Women with more childbirths had a reduced risk of BC. In premenopausal women, $2+$ childbirths were associated with a $20 \%$ reduced risk of BC (childbirths: 2 vs. $0, \mathrm{OR}, 0.80 ; 95 \% \mathrm{Cl}, 0.65$ to 0.97 ; childbirths: $3+$ vs. $1, \mathrm{OR}$, $0.81 ; 95 \% \mathrm{Cl}, 0.70$ to 0.94$)$. Postmenopausal women with 2 childbirths had a $40 \%$ reduced risk of BC and those with 3 or more childbirths had a much more strongly reduced risk (childbirths: $3+$ vs. $0, \mathrm{OR}, 0.40 ; 95 \% \mathrm{Cl}, 0.35$ to 0.43 ; $p$-heterogeneity between premenopausal and postmenopausal wom- 
Table 1. BC risk associated with parity, number of childbirths, and breastfeeding and its duration among BC cases and population-based matched controls in the Korea Breast Cancer Study, 2004 to 2012

\begin{tabular}{|c|c|c|c|c|c|c|c|c|c|}
\hline & & Total wom & & Pre & enopausal & women & Post & nenopausa & women \\
\hline & $\begin{array}{c}\text { BC cases } \\
\text { n (\%) }\end{array}$ & $\begin{array}{c}\text { Controls } \\
\text { n (\%) }\end{array}$ & OR (95\% CI) & $\begin{array}{c}\text { BC cases } \\
\text { n (\%) }\end{array}$ & $\begin{array}{c}\text { Controls } \\
\text { n (\%) }\end{array}$ & OR $(95 \% \mathrm{CI})$ & $\begin{array}{c}\text { BC cases } \\
\text { n (\%) }\end{array}$ & $\begin{array}{c}\text { Controls } \\
\text { n (\%) }\end{array}$ & OR $(95 \% \mathrm{CI})$ \\
\hline Parity & & & & & & & & & \\
\hline Nullipara & $291(2.3)$ & $144(1.1)$ & $1.35(1.06,1.69)$ & $152(2.4)$ & $94(1.5)$ & $1.16(0.86-1.59)$ & 139 (2.12) & $50(0.8)$ & $1.72(1.18,2.50)$ \\
\hline Para & 12598 (97.7) & 12745 (98.9) & 1.00 (reference) & 6170 (97.6) & 6228 (98.5) & 1.00 (reference) & 6428 (97.9) & 6517 (99.2) & 1.00 (reference) \\
\hline No. of childbirt & & & & & & & & & \\
\hline 0 & $291(2.3)$ & $144(1.1)$ & 1.00 (reference) & $152(2.4)$ & $94(1.5)$ & 1.00 (reference) & $139(2.1)$ & $50(0.8)$ & 1.00 (reference) \\
\hline 1 & 2046 (15.9) & $1370(10.6)$ & $0.88(0.69,1.14)$ & $1200(19.0)$ & $853(13.5)$ & $1.01(0.74,1.39)$ & 846 (12.9) & $517(7.9)$ & $0.72(0.48,1.06)$ \\
\hline 2 & 7461 (57. 9) & 7552 (58.6) & $0.71(0.61,0.83)$ & 4248 (67.2) & $4526(71.6)$ & $0.80(0.65,0.97)$ & $3213(48.9)$ & $3026(46.1)$ & $0.58(0.45,0.74)$ \\
\hline $3+$ & $3091(24.0)$ & $3823(29.7)$ & $0.62(0.54,0.80)$ & 722 (11.4) & $849(13.4)$ & $0.81(0.70,0.94)^{1}$ & $2369(36.1)$ & $2974(45.3)$ & $0.40(0.35,0.43)^{1}$ \\
\hline Breastfeeding & & & & & & & & & \\
\hline No children & $291(2.3)$ & $144(1.1)$ & $1.38(1.10,1.75)$ & $152(2.4)$ & $94(1.5)$ & $1.17(0.86,1.58)$ & $139(2.1)$ & $50(0.8)$ & $1.80(1.24,2.62)$ \\
\hline Never & $2966(23.0)$ & 1975 (15.3) & 1.00 (reference) & 1945 (30.8) & $1340(21.2)$ & 1.00 (reference) & $1021(15.6)$ & 635 (9. 7) & 1.00 (reference) \\
\hline Ever & $9632(74.7)$ & 10770 (83.6) & $0.60(0.55,0.64)$ & 4225 (66.8) & 4888 (77.3) & $0.59(0.54,0.65)$ & 5407 (82.3) & 5882 (89.6) & $0.60(0.53,0.68)$ \\
\hline Breastfeeding & & & & & & & & & \\
\hline 0 & 3257 (25.3) & $2119(16.4)$ & 1.00 (reference) & 2097 (33.2) & $1434(22.7)$ & 1.00 (reference) & $1160(17.7)$ & $685(10.4)$ & 1.00 (reference) \\
\hline $1-12$ & 4116 (31.9) & $3624(28.1)$ & $0.77(0.71,0.84)$ & 2372 (37.5) & $2470(39.1)$ & $0.67(0.60,0.74)^{1}$ & $1744(26.6)$ & $1154(17.6)$ & $0.99(0.86,1.14)^{\prime}$ \\
\hline $13-24$ & $2901(22.5)$ & $3576(27.7)$ & $0.57(0.52,0.62)$ & $1293(20.5)$ & $1711(27.1)$ & $0.55(0.48,0.62)$ & $1608(24.5)$ & 1865 (28.4) & $0.60(0.52,0.70)$ \\
\hline $25+$ & $1973(15.3)$ & 3002 (23.3) & $0.41(0.37,0.46)$ & $398(6.3)$ & $599(9.5)$ & $0.44(0.37,0.53)$ & $1575(24.0)$ & $2403(36.6)$ & $0.44(0.38,0.52)$ \\
\hline
\end{tabular}

The $\mathrm{OR}(95 \% \mathrm{CI})$ in all women was estimated in conditional logistic regression stratified by age and enrollment year and adjusted for family history of $\mathrm{BC}$, age at menarche, age at first full-term pregnancy, duration of oral contraceptive use, BMI, and breastfeeding duration in the analyses of parity and number of childbirths; or adjusted for family history of $\mathrm{BC}$, age at menarche, age at first full-term pregnancy, duration of oral contraceptive use, BMI, and number of childbirths in the analyses of breastfeeding and its duration. The OR $(95 \% \mathrm{Cl})$ in premenopausal and postmenopausal women was estimated in an unconditional logistic regression model stratified by age and year and adjusted for same covariates.

$\mathrm{BC}$, breast cancer; $\mathrm{OR}$, odds ratio; $\mathrm{Cl}$, confidence interval; $\mathrm{BMI}$, body mass index.

' $p$-value for the heterogeneity of two ORs (95\% Cls) from 2 subgroups classified by menopausal status: $p<0.001$ for [childbirths $=3+$ ]; $p<0.001$ for [breastfeeding $=1-12$ months].

Table 2. BC risk associated with number of childbirths and breastfeeding duration according to HR status among BC cases and population-based matched controls in the Korea Breast Cancer Study, 2004 to 2012

\begin{tabular}{|c|c|c|c|c|c|c|}
\hline & \multicolumn{2}{|c|}{ Total women } & \multicolumn{2}{|c|}{ Premenopausal women } & \multicolumn{2}{|c|}{ Postmenopausal women } \\
\hline & HR+ & HR- & HR+ & HR- & HR+ & HR- \\
\hline \multicolumn{7}{|c|}{ No. of childbirths } \\
\hline 1 & $0.78(0.61,0.99)$ & $1.25(0.87,1.79)$ & $0.94(0.69,1.30)^{2}$ & $1.64(0.96,2.78)^{2}$ & $0.57(0.39,0.85)^{2}$ & $0.88(0.53,1.47)^{2}$ \\
\hline 2 & $0.52(0.44,0.60)^{1}$ & $1.04(0.83,1.30)^{1}$ & $0.62(0.50,0.78)^{2,3}$ & $1.31(0.92,1.86)^{2}$ & $0.48(0.37,0.61)^{2,3}$ & $0.78(0.57,1.07)^{2}$ \\
\hline $3+$ & $0.39(0.34,0.46)^{1}$ & $1.05(0.86,1.18)^{1}$ & $0.55(0.48,0.61)^{2}$ & $1.39(0.99,1.83)^{2}$ & $0.22(0.18,0.25)^{2}$ & $0.73(0.57,0.99)^{2}$ \\
\hline 0 & 1.00 (reference) & 1.00 (reference) & 1.00 (reference) & 1.00 (reference) & 1.00 (reference) & 1.00 (reference) \\
\hline $1-12$ & $0.74(0.69,0.80)$ & $0.77(0.68,0.87)$ & $0.67(0.61,0.73)^{2.3}$ & $0.65(0.55,0.77)^{2}$ & $1.03(0.89,1.20)^{2,3}$ & $0.94(0.78,1.13)^{2}$ \\
\hline $13-24$ & $0.52(0.48,0.57)$ & $0.52(0.46,0.60)$ & $0.53(0.48,0.59)$ & $0.48(0.39,0.58)$ & $0.64(0.55,0.74)$ & $0.55(0.46,0.67)$ \\
\hline $25+$ & $0.40(0.37,0.44)$ & $0.40(0.34,0.47)$ & $0.45(0.38,0.52)$ & $0.49(0.37,0.66)$ & $0.48(0.41,0.57)$ & $0.40(0.32,0.49)$ \\
\hline
\end{tabular}

Values are presented as OR (95\% Cl).

The $\mathrm{OR}(95 \% \mathrm{Cl})$ in all women was estimated in conditional logistic regression stratified by age and enrollment year and adjusted for family history of $\mathrm{BC}$, age at menarche, age at first full-term pregnancy, duration of oral contraceptive use, BMI, and breastfeeding duration in the analyses of parity and number of childbirths; or adjusted for family history of $\mathrm{BC}$, age at menarche, age at first full-term pregnancy, duration of oral contraceptive use, BMI, and number of childbirths in the analyses of breastfeeding and its duration. $\mathrm{HR}$, hormone receptor; $\mathrm{BC}$, breast cancer; $\mathrm{OR}$, odds ratio; $\mathrm{Cl}$, confidence interval; $\mathrm{BMI}$, body mass index.

' $p$-value for the heterogeneity of two ORs (95\% Cls) from HR+ subgroup and HR- subgroup in total women: $<0.001$ for [childbirths $=2$ ]; $<0.001$ for [childbirths $=3+$ ] . ${ }^{2} p$-value for the heterogeneity of four ORs ( $95 \% \mathrm{Cls}$ ) from 4 subgroups classified by HR and menopausal status: 0.017 for [childbirths $=1$ ]; $<0.001$ for [childbirths $=2$ ]; $<0.001$ for [childbirths $=3+$ ]; $<0.001$ for [breastfeeding $=1-12$ months].

${ }^{3} p$-value for the heterogeneity of two ORs (95\% Cls) from 2 subgroups classified by menopausal status in HR+ groups $=0.134$ for [childbirths $=2$ ]; $<0.001$ for [breastfeeding $=1-12$ months]. 

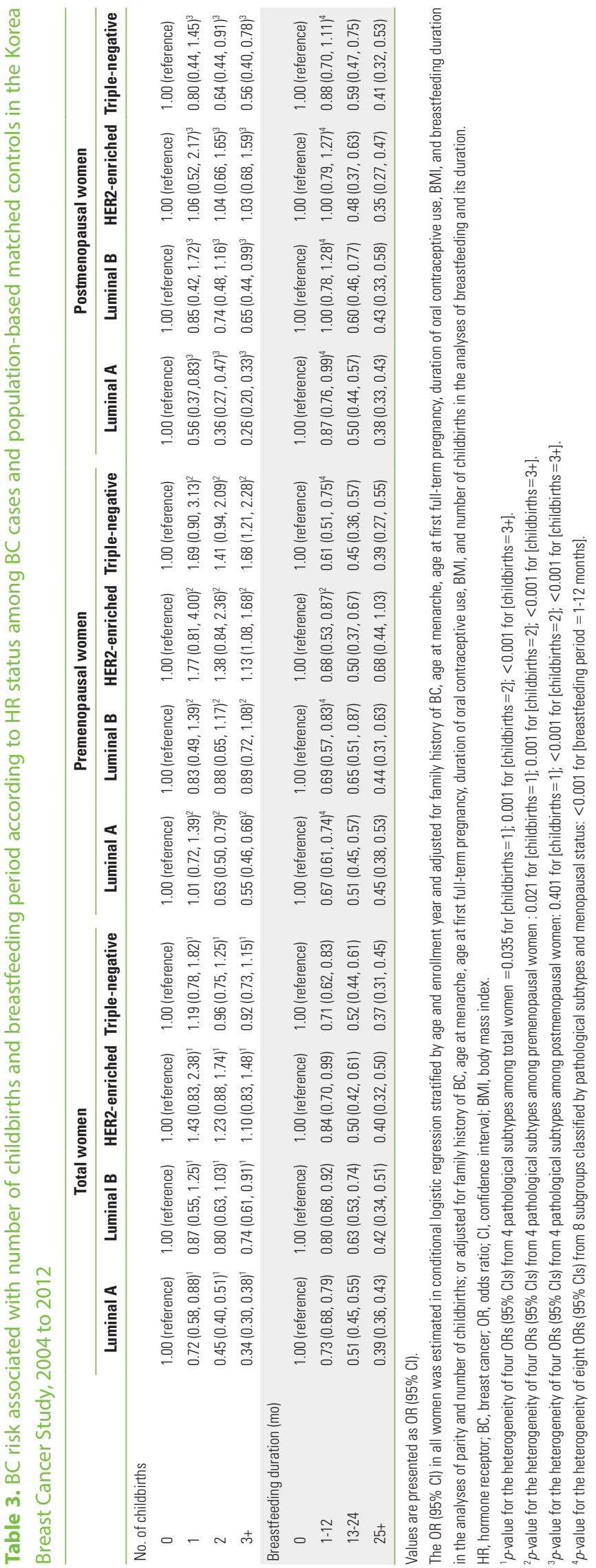

Table 4. BC risk associated with the combined effect of number of childbirths and breastfeeding duration in the Korea Breast Cancer Study, 2004 to 2012

\begin{tabular}{lccc}
\hline $\begin{array}{c}\text { No. of } \\
\text { childbirths }\end{array}$ & $\begin{array}{c}\text { Breastfeeding } \\
\text { duration (mo) }\end{array}$ & $\begin{array}{c}\text { Premenopausal } \\
\text { women }\end{array}$ & $\begin{array}{c}\text { Postmenopausal } \\
\text { women }\end{array}$ \\
\hline 0 & 0 & 1.00 (reference) & 1.00 (reference) \\
1 & 0 & $0.98(0.70,1.39)$ & $0.65(0.43,0.99)$ \\
2 & 0 & $0.78(0.69,0.92)$ & $0.63(0.54,0.73)$ \\
$3+$ & 0 & $0.89(0.86,0.94)^{1}$ & $0.47(0.44,0.50)^{1}$ \\
1 & $1-12$ & $0.65(0.58,0.74)$ & $0.64(0.55,0.74)$ \\
1 & $13-24$ & $0.69(0.51,0.60)$ & $0.49(0.42,0.58)$ \\
2 & $1-12$ & $0.52(0.45,0.61)$ & $0.52(0.43,0.62)$ \\
$3+$ & $1-12$ & $0.63(0.59,0.68)$ & $0.70(0.61,0.80)$ \\
2 & $13-24$ & $0.44(0.39,0.51)$ & $0.36(0.30,0.44)$ \\
2 & $25+$ & $0.36(0.34,0.39)^{1}$ & $0.27(0.23,0.32)^{1}$ \\
$3+$ & $13-24$ & $0.36(0.35,0.38)^{1}$ & $0.29(0.25,0.33)^{1}$ \\
$3+$ & $25+$ & $0.34(0.32,0.38)^{1}$ & $0.21(0.18,0.26)^{1}$ \\
\hline
\end{tabular}

Values are presented as OR (95\% CI).

The $\mathrm{OR}(95 \% \mathrm{Cl})$ in all women was estimated in conditional logistic regression stratified by age and enrollment year and adjusted for family history of $\mathrm{BC}$, age at menarche, age at first full-term pregnancy, duration of oral contraceptive use, BMI, and breastfeeding duration in the analyses of parity and number of childbirths; or adjusted for family history of $\mathrm{BC}$, age at menarche, age at first full-term pregnancy, duration of oral contraceptive use, BMI, and number of childbirths in the analyses of breastfeeding and its duration. The calculated actual $\mathrm{OR}$ values in premenopausal and postmenopausal women was estimated using an unconditional logistic regression model stratified by age and year and adjusted for same covariates. $\mathrm{BC}$, breast cancer, $\mathrm{OR}$, odds ratio; $\mathrm{Cl}$, confidence interval; $\mathrm{BMI}$, body mass index. ${ }^{1} p$-value for the heterogeneity of two ORs $(95 \% \mathrm{Cls})$ from 2 subgroups classified by menopausal status: $<0.001$ for [children $=3+$ and never breastfeeding] 0.005 for [children $=3+$ and breastfeeding $=13-24$ months]; 0.002 for [children $=2$ and breastfeeding $=25+$ months] $;<0.001$ for [children $=3+$ and breastfeeding $=25+$ months] .

en $<0.001$ ). This effect was much stronger for $\mathrm{HR}+\mathrm{BC}$ ( $p$-heterogeneity between $\mathrm{HR}+$ and $\mathrm{HR}-<0.001,0.009,<0.001$ in total, premenopausal, and postmenopausal women, respectively) (childbirths: $3+$ vs. $0,0 R, 0.55 ; 95 \% \mathrm{Cl}, 0.48$ to 0.61 ; and $\mathrm{OR}, 0.22 ; 95 \% \mathrm{Cl}, 0.18$ to 0.25 in premenopausal and postmenopausal women, respectively) and in luminal A BC ( $p$-heterogeneity among the $4 \mathrm{BC}$ subtypes in total, premenopausal, and postmenopausal women <0.001) (childbirths: $3+$ vs. 0 , $\mathrm{OR}, 0.55 ; 95 \% \mathrm{Cl}, 0.46$ to 0.66 ; and $\mathrm{OR}, 0.26 ; 95 \% \mathrm{Cl}, 0.20$ to 0.33 in premenopausal and postmenopausal women, respectively). In particular, postmenopausal women with 3 or more childbirths showed a $44 \%$ reduced risk of TNBC (childbirths: $3+\mathrm{vs.}$ $0, \mathrm{OR}, 0.56 ; 95 \% \mathrm{Cl}, 0.40$ to 0.78 ). Likewise, longer breastfeeding was associated with a reduced risk of $B C$. Relative to women with no breastfeeding, premenopausal women with a breastfeeding duration of 1-12 months had a reduced risk of $\mathrm{BC}(\mathrm{OR}, 0.67 ; 95 \% \mathrm{Cl}, 0.60$ to 0.74$)$, while postmenopausal women with the same breastfeeding duration had no reduced risk for BC ( $p$-heterogeneity<0.001). Women with a much lon- 


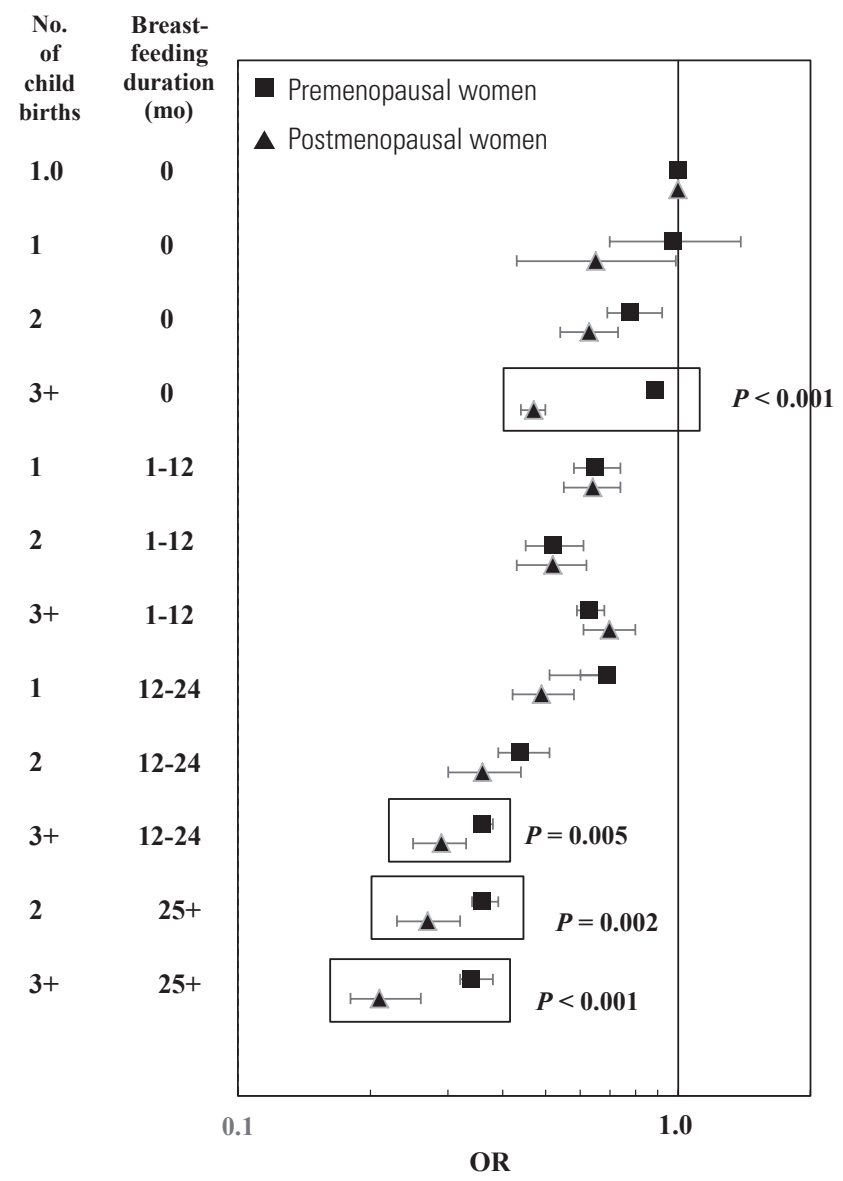

Figure 1. Heterogeneous effect on breast cancer risk by the combination of number of childbirths and breastfeeding duration in all breast cancer cases and population-based matched controls, the Korea Breast Cancer Study, 2004 to 2012. P, p-heterogeneity; OR, odds ratio.

ger breastfeeding duration ( $25+$ months) had a $56 \%$ reduced risk for $\mathrm{BC}$, regardless of menopausal status $(\mathrm{OR}, 0.44 ; 95 \% \mathrm{Cl}$, 0.37 to 0.53 ; and $\mathrm{OR}, 0.44 ; 95 \% \mathrm{Cl}, 0.38$ to 0.52 in premenopausal and postmenopausal women, respectively). The protective effect of breastfeeding on BC risk was not significantly different according to HR status or pathological subtype.

The combined effect of breastfeeding and childbirth is shown in Figure 1 and Table 4. Since childbirth and breastfeeding were events in premenopausal women, we described to focus on the $\mathrm{BC}$ risk in premenopausal women. Compared to $\mathrm{BC}$ risk in women with no-childbirth and never-breastfeeding, there were no $\mathrm{BC}$ risk reduction in women with 1 childbirth but never-breastfeeding. However, the $\mathrm{BC}$ risk in women with $2+$ childbirths but never-breastfeeding began to decline and their $\mathrm{BC}$ risk was reduced by at least 0.89 -fold ( $3+$ childbirths: OR, $0.8995 \% \mathrm{Cl}, 0.86$ to $0.94 ; 2$ childbirths: $\mathrm{OR}, 0.78 ; 95 \% \mathrm{Cl}$,
0.69 to 0.92 ). The $B C$ risk was reduced by at least 0.69 -fold for women with 1 childbirth and breastfeeding for $\geq 1$ month (breastfeeding for 1-12 months: OR, $0.65 ; 95 \% \mathrm{Cl}, 0.58$ to 0.74 ; breastfeeding for $\geq 13$ months: $\mathrm{OR}, 0.69 ; 95 \% \mathrm{Cl}, 0.51$ to 0.60 ), at least 0.63 -fold for women with $2+$ childbirth and breastfeeding for 1-12 months ( 2 childbirths: OR, $0.52 ; 95 \% \mathrm{Cl}, 0.45$ to $0.61 ; 3+$ childbirths: $\mathrm{OR}, 0.63 ; 95 \% \mathrm{Cl}, 0.59$ to 0.68 ), and at least 0.44 -fold for women with $2+$ childbirth and breastfeeding for $\geq 13$ months ( 2 childbirths and breastfeeding for 13-24 months: OR, 0.44; 2 childbirths and breastfeeding for 13-24 months: OR, 0.36; 3+ childbirths and breastfeeding for 13-24 months: OR, 0.36; 3+ childbirths and breastfeeding for $\geq 25$ months: OR, 0.34). The combined OR of never breastfeeding and $3+$ childbirths was different between premenopausal and postmenopausal women ( $p$-heterogeneity $<0.001$ ). Additionally, the combined OR of a breastfeeding duration of $25+$ months and 2+ childbirths was different between premenopausal and postmenopausal women ( $p$-heterogeneity $=0.002$, $<0.001$ for 2 and $3+$ childbirths, respectively).

The patients analyzed in this study had the following distribution of BC stages: stage $0,5.3 \%$; stage I, $18.9 \%$; stage II, $17.7 \%$; stage III, $6.5 \%$; and stage IV, $0.7 \%$. The $B C$ risk associated with number of childbirths, breastfeeding, and their combination by stage (0-1, 2, and $3+$ ) was not heterogeneous (data not shown).

\section{DISCUSSION}

In our study, individual risk factors such as childbirth and breastfeeding were associated with a reduced risk for $\mathrm{BC}$. The effect of giving birth on $\mathrm{BC}$ risk reduction was heterogeneous between premenopausal and postmenopausal women, and a much stronger association was found in postmenopausal women (childbirths of $3+$ vs. 0: 20 and 60\% risk reduction in premenopausal and postmenopausal women, respectively). A breastfeeding duration of 1-12 months, relative to never breastfeeding, was associated with a strongly decreased BC risk in premenopausal women, but no such association was found in postmenopausal women. The longer breastfeeding durations of 13-24 and 25+ months were associated with a further decrease in the risk for $\mathrm{BC}$, but the effect was not different by menopausal status. $\mathrm{BC}$ risk reduction by 3 or more childbirths was strongly observed in $\mathrm{HR}+\mathrm{BC}$, as well as in the luminal $A$ subtype. By contrast, the reduction of $B C$ risk by a longer breastfeeding period did not vary according to $\mathrm{HR}$ or 
pathological subtype. The combined effect of childbirth and breastfeeding on $\mathrm{BC}$ risk reduction showed a multiplicative synergistic effect. The combination of both factors had a heterogeneous association between premenopausal and postmenopausal women who had breastfed for $25+$ months or had $3+$ childbirths. Previous studies on the individual effect of childbirth on BC risk showed a significantly decreased risk, with a much larger reduction in postmenopausal women $[1,3,22]$. A consistent relationship between breastfeeding and postmenopausal status has not been found $[3,4,6,8]$ The recent meta-analyses of the relationship between breastfeeding and $\mathrm{BC}$ risk confirmed an inverse association with breastfeeding itself and found that a greater reduction of $\mathrm{BC}$ risk was associated with longer breastfeeding $[8,16,23]$.

Few previous studies have investigated the heterogeneous associations of childbirth and breastfeeding with $\mathrm{BC}$ risk, and their results were controversial. Two case-control studies of breastfeeding itself and longer breastfeeding periods showed a reduced $B C$ risk in only premenopausal women $[5,7]$. Those previous results are similar to our results. However, our results showed some differences according to breastfeeding duration. When the breastfeeding period was 1-12 months, only premenopausal women had a risk reduction in $\mathrm{BC}$, which was a heterogeneous association. A previous cohort study of childbirth showed a significant reduction of BC risk only in postmenopausal women [24], whereas our findings also showed a heterogeneously lower risk for premenopausal women than postmenopausal women, but a reduction in $\mathrm{BC}$ risk associated with 3 or more childbirths was observed in all women, regardless of menopausal status. Our study reported more detailed results than previous studies because the number of subjects in our study is the largest among the studies that have been conducted in this field.

Our findings of a heterogeneous association with childhood according to HR status (a stronger association in $\mathrm{HR}+\mathrm{BC}$ ) and a non-heterogeneous association of breastfeeding according to HR status have consistently been observed in individual studies (for childbirth: [25-27]; for breastfeeding: [27]) and review papers $[3,28]$. However, our findings of a heterogeneous association with childbirth according to the pathological subtype and a non-heterogeneous association with breastfeeding do not correspond with those of previous studies. In our study, a greater reduction in $\mathrm{BC}$ risk was shown with more childbirths in all 4 subtypes, with a particularly strong trend for the luminal A subtype, whereas previous studies reported inconsistent results, such as a heterogeneous association between the luminal A and B subtypes (inverse association) and the HER2/ TNBC subtypes (no significant association) $[26,29]$ or a nonheterogeneous association with all subtypes [27]. The finding that $B C$ risk was reduced with breastfeeding in all 4 subtypes and non-heterogeneously associated with the 4 subtypes is not concordant with the findings of previous studies [26-30]. Findings of the lowest risk in the luminal A subtype relative to other subtypes agree with our observation of a strongly lower risk in $\mathrm{HR}+\mathrm{BC}$ than in $\mathrm{HR}-\mathrm{BC}$. Previous studies showed various results, such as a significantly reduced risk of luminal $A$ and B BC [30], a reduced risk of TNBC $[26,28,30]$, a non-significant association with HER2-expressing $B C[26,28]$, and a nonsignificant association with all subtypes [27].

Although a combined effect of breastfeeding and giving birth on BC risk reduction was not reported in previous studies, a synergistic multiplicative effect was presented in this paper, as the $B C$ risk reduction was higher with an increase in both factors (i.e., more childbirths and a longer breastfeeding duration). It is biologically plausible that the presence of both factors induces a much shorter duration of exposure to hormones, in accordance with the estrogen hypothesis, in addition to the biochemical mechanisms associated with each protective factor $[14,15]$. In particular, this combined effect was not different across menopausal status, HR, or pathological subtype.

This study has several limitations. First, lifestyle factors well known as risk factors for BC (such as physical exercise [31], smoking and drinking [32,33], and food intake [34-37]) were not included. Second, it can be suspected that there is a recall bias in women's response of breastfeeding duration because the response to the total breastfeeding period of women was concentrated in a particular month (such 12-month, 24-month, 36-month by an interval of 12-month). The strength of our research is that it incorporated large-scale data from across the country. In Korea, a total of 115928 new cases of BC occurred during the 9 years from 2004 to 2012 [38]. About $11 \%$ of these epidemiologic data were used in this study. In addition, participants were matched by age and year of enrollment to improve the comparability by controlling for the age effect and the birth effect.

In conclusion, the effect of childbirth on $\mathrm{BC}$ risk reduction was heterogeneous according to menopausal status, and the $\mathrm{BC}$ risk reduction associated with 3 or more childbirths was much stronger in postmenopausal women than in premeno- 
pausal women. By contrast, longer breastfeeding durations of $13+$ months were non-heterogeneously associated with menopausal status, but only women with a breastfeeding duration of 1-12 months showed heterogeneity according to menopausal status, with only premenopausal women showing a significant $B C$ risk reduction. The association of breastfeeding and its duration on $\mathrm{BC}$ risk was not different according to HR status or pathological subtype. More childbirths and longer breastfeeding exerted a combined effect on BC risk reduction. The heterogeneous association of the combination of breastfeeding (1-12 months) and any childbirths on $\mathrm{BC}$ risk by menopausal status was only due to the heterogeneous association of breastfeeding according to menopause, not the combined effect. The combined effects of women's childbirth and breastfeeding on $\mathrm{BC}$ risk reduction are summarized, focusing on premenopausal women because birth and breastfeeding are important life events of premenopausal women who can only be modifiable in premenopausal women. Compared to $\mathrm{BC}$ risk in women with no-childbirth and never-breastfeeding, a woman who have at least one birth can only benefit from $B C$ risk reduction if she experiences breastfeeding, while she does not do breastfeeding she does not have risk reduction effect. The BC risk is decreased by $10-20 \%$ for women with $2+$ childbirth but never-breastfeeding, about 30\% for women with 1 childbirth but breastfeeding for at least 1 months, 40-50\% for women with 2+ childbirth but breastfeeding for 1-12 months, and $>60 \%$ for women with $2+$ childbirth but breastfeeding for $\geq 13$ months.

In the view of the population-attributable fraction (PAF), two previous Korean papers suggest that the PAF of breastfeeding and/or pregnancy (including age at first birth) is estimated to be $7-11 \%$ for Korean women $B C[30,39]$. If women with $<2$ childbirths and $<13$ months of breastfeeding are considered to be the high-risk group, the PAF for BC prevention through interventions in the high-risk group would be estimated to be about $7.5 \%$ according to our data, which is similar to the prior PAF value estimated in prior studies. The PAF (7.5\%) associated with the high-risk group defined by the combination of childbirth and breastfeeding period accounts for about one-fourth of the PAF (33\%) from all modifiable factors [30]. Since the fertility rate in Korea has rapidly decreased and still not recovered (6.0 births per fertile woman in 1960; 1.08 in 2005 [the lowest]; 1.17 in 2016; Korean Statistical Information Service [http://kosis.kr]), the relative impact of these factors on breast cancer prevention seems to be more important than in the past. Al- though low childbirth and/or the combination of low childbirth and short breastfeeding can considered to make a partial contribution to $B C$ in Korea, this paper is expected to serve as scientific evidence to support national cancer management policies for BC.

Our findings regarding $\mathrm{BC}$ risk modification by the combination of childbirth and breastfeeding and the full evaluation of heterogeneous associations by menopausal status, HR, and pathological subtypes are the first such findings worldwide, to the best of our knowledge. This study may provide a scientific basis for national cancer management policies involving the promotion of childbirth and breastfeeding, although childbirth and breastfeeding contribute only partially to the development of BC.

\section{ACKNOWLEDGEMENTS}

This study was partially supported by a grant from the National R\&D Program for Cancer Control and the Korea Health Technology R\&D Project through the Korea Health Industry Development Institute (KHIDI), Ministry of Health and Welfare, Republic of Korea (1420190; HI16C1127).

We would like to express our sincere appreciation to the all members of the National Breast Cancer Society who participated in the Breast Cancer Registration Program of the Korean Breast Cancer Society; and to all investigators who participated in cohort enrollment and data collection, as well as those who worked at the headquarters of the Korean Genomic and Epidemiologic Study (KoGES), Korea Centers for Disease Control and Prevention.

\section{CONFLICT OF INTEREST}

The authors have no conflicts of interest associated with the material in this paper.

\section{ORCID}

Seok Hun Jeong https://orcid.org/0000-0002-5954-5369

Yoonsuk An https://orcid.org/0000-0002-2499-2281

Boyoung Park https://orcid.org/0000-0003-1902-3184

Daehee Kang https://orcid.org/0000-0003-4031-5878

Wonshik Han https://orcid.org/0000-0001-7310-0764

Keun-Young Yoo https://orcid.org/0000-0002-3726-5390

Sue K. Park https://orcid.org/0000-0001-5002-9707 


\section{REFERENCES}

1. Reeves GK, Pirie K, Green J, Bull D, Beral V; Million Women Study Collaborators. Reproductive factors and specific histological types of breast cancer: prospective study and metaanalysis. Br J Cancer 2009;100(3):538-544.

2. Hinkula M, Pukkala E, Kyyrönen P, Kauppila A. Grand multiparity and the risk of breast cancer: population-based study in Finland. Cancer Causes Control 2001;12(6):491-500.

3. Ma H, Bernstein L, Pike MC, Ursin G. Reproductive factors and breast cancer risk according to joint estrogen and progesterone receptor status: a meta-analysis of epidemiological studies. Breast Cancer Res 2006;8(4):R43.

4. Islam T, Matsuo $\mathrm{K}$, Ito $\mathrm{H}$, Hosono $\mathrm{S}$, Watanabe $\mathrm{M}$, Iwata $\mathrm{H}$, et al. Reproductive and hormonal risk factors for luminal, HER2overexpressing, and triple-negative breast cancer in Japanese women. Ann Oncol 2012;23(9):2435-2441.

5. Newcomb PA, Storer BE, Longnecker MP, Mittendorf R, Greenberg ER, Clapp RW, et al. Lactation and a reduced risk of premenopausal breast cancer. N Engl J Med 1994;330(2):81-87.

6. Bernier MO, Plu-Bureau G, Bossard N, Ayzac L, Thalabard JC. Breastfeeding and risk of breast cancer: a metaanalysis of published studies. Hum Reprod Update 2000;6(4):374-386.

7. Martin RM, Middleton N, Gunnell D, Owen CG, Smith GD. Breast-feeding and cancer: the Boyd Orr cohort and a systematic review with meta-analysis. J Natl Cancer Inst 2005;97 (19):1446-1457.

8. Unar-Munguía M, Torres-Mejía G, Colchero MA, González de Cosío T. Breastfeeding mode and risk of breast cancer: a doseresponse meta-analysis. J Hum Lact 2017;33(2):422-434.

9. Freund C, Mirabel L, Annane K, Mathelin C. Breastfeeding and breast cancer. Gynecol Obstet Fertil 2005;33(10):739-744 (French).

10. Russo J, Russo IH. Toward a physiological approach to breast cancer prevention. Cancer Epidemiol Biomarkers Prev 1994;3(4):353-364.

11. Russo J, Mailo D, Hu YF, Balogh G, Sheriff F, Russo IH. Breast differentiation and its implication in cancer prevention. Clin Cancer Res 2005;11(2 Pt 2):931s-936s.

12. Russo J, Moral R, Balogh GA, Mailo D, Russo IH. The protective role of pregnancy in breast cancer. Breast Cancer Res 2005; 7(3):131-142.

13. Bernstein L. Epidemiology of endocrine-related risk factors for breast cancer. J Mammary Gland Biol Neoplasia 2002;7(1):315.
14. McNeilly AS, Tay CC, Glasier A. Physiological mechanisms underlying lactational amenorrhea. Ann N Y Acad Sci 1994;709: 145-155.

15. Henderson BE, Ross RK, Judd HL, Krailo MD, Pike MC. Do regular ovulatory cycles increase breast cancer risk? Cancer 1985; 56(5):1206-1208.

16. Zhou Y, Chen J, Li Q, Huang W, Lan H, Jiang H. Association between breastfeeding and breast cancer risk: evidence from a meta-analysis. Breastfeed Med 2015;10(3):175-182.

17. Sung HK, Ma SH, Choi JY, Hwang Y, Ahn C, Kim BG, et al. The effect of breastfeeding duration and parity on the risk of epithelial ovarian cancer: a systematic review and meta-analysis. J Prev Med Public Health 2016:49(6):349-366.

18. Ahn SH, Son BH, Kim SW, Kim SI, Jeong J, Ko SS, et al. Poor outcome of hormone receptor-positive breast cancer at very young age is due to tamoxifen resistance: nationwide survival data in Korea: a report from the Korean Breast Cancer Society. J Clin Oncol 2007;25(17):2360-2368.

19. Kim Y, Han BG; KoGES Group. Cohort profile: the Korean genome and epidemiology study (KoGES) consortium. Int J Epidemiol 2017;46(2):e20.

20. Woodward M. Epidemiology: study design and data analysis. Boca Raton: CRC Press; 2014, p. 149-161.

21. Stuart EA. Matching methods for causal inference: a review and a look forward. Stat Sci 2010;25(1):1-21.

22. Ma H, Bernstein L, Pike MC, Ursin G. Reproductive factors and breast cancer risk according to joint estrogen and progesterone receptor status: a meta-analysis of epidemiological studies. Breast Cancer Res 2006;8(4):R43.

23. Anothaisintawee T, Wiratkapun C, Lerdsitthichai P, Kasamesup $\mathrm{V}$, Wongwaisayawan S, Srinakarin J, et al. Risk factors of breast cancer: a systematic review and meta-analysis. Asia Pac J Public Health 2013;25(5):368-387.

24. Clavel-Chapelon F; E3N-EPIC Group. Differential effects of reproductive factors on the risk of pre- and postmenopausal breast cancer. Results from a large cohort of French women. Br J Cancer 2002;86(5):723-727.

25. Ursin G, Bernstein L, Lord SJ, Karim R, Deapen D, Press MF, et al. Reproductive factors and subtypes of breast cancer defined by hormone receptor and histology. Br J Cancer 2005;93 (3):364-371.

26. Ellingjord-Dale M, Vos L, Tretli S, Hofvind S, Dos-Santos-Silva I, Ursin G. Parity, hormones and breast cancer subtypes: results from a large nested case-control study in a national screening program. Breast Cancer Res 2017;19(1):10. 
27. Ritte R, Tikk K, Lukanova A, Tjønneland A, Olsen A, Overvad K, et al. Reproductive factors and risk of hormone receptor positive and negative breast cancer: a cohort study. BMC Cancer 2013;13:584.

28. Anderson KN, Schwab RB, Martinez ME. Reproductive risk factors and breast cancer subtypes: a review of the literature. Breast Cancer Res Treat 2014;144(1):1-10.

29. Lambertini M, Santoro L, Del Mastro L, Nguyen B, Livraghi L, Ugolini $D$, et al. Reproductive behaviors and risk of developing breast cancer according to tumor subtype: a systematic review and meta-analysis of epidemiological studies. Cancer Treat Rev 2016;49:65-76.

30. Park B, Choi JY, Sung HK, Ahn C, Hwang Y, Jang J, et al. Attribution to heterogeneous risk factors for breast cancer subtypes based on hormone receptor and human epidermal growth factor 2 receptor expression in Korea. Medicine (Baltimore) 2016;95(14):e3063.

31. Wu Y, Zhang D, Kang S. Physical activity and risk of breast cancer: a meta-analysis of prospective studies. Breast Cancer Res Treat 2013;137(3):869-882.

32. Gaudet MM, Gapstur SM, Sun J, Diver WR, Hannan LM, Thun MJ. Active smoking and breast cancer risk: original cohort data and meta-analysis. J Natl Cancer Inst 2013;105(8):515525.

33. Seitz HK, Pelucchi C, Bagnardi V, La Vecchia C. Epidemiology and pathophysiology of alcohol and breast cancer: update 2012. Alcohol Alcohol 2012;47(3):204-212.

34. Gandini S, Merzenich H, Robertson C, Boyle P. Meta-analysis of studies on breast cancer risk and diet: the role of fruit and vegetable consumption and the intake of associated micronutrients. Eur J Cancer 2000;36(5):636-646.

35. Bae JM. Reinterpretation of the results of a pooled analysis of dietary carotenoid intake and breast cancer risk by using the interval collapsing method. Epidemiol Health 2016;38:e2016024.

36. Ko KP, Kim SW, Ma SH, Park B, Ahn Y, Lee JW, et al. Dietary intake and breast cancer among carriers and noncarriers of BRCA mutations in the Korean Hereditary Breast Cancer Study. Am J Clin Nutr 2013;98(6):1493-1501.

37. Boyd NF, Stone J, Vogt KN, Connelly BS, Martin LJ, Minkin S. Dietary fat and breast cancer risk revisited: a meta-analysis of the published literature. Br J Cancer 2003;89(9):1672-1685.

38. Korean Statistical Information Service. Cancer registration statistics (1999-2014); 2016 [cited 2017 Nov 23]. Available from: http:// kosis.kr/statHtml/statHtml.do?orgld=117\&tblld=DT_117N_ A00023\&conn_path $=12$ (Korean).

39. Park B, Park S, Shin HR, Shin A, Yeo Y, Choi JY, et al. Populationattributable risks of modifiable reproductive factors for breast and ovarian cancers in Korea. BMC Cancer 2016;16:5. 